\title{
Early-Stage Lung Cancer Detection Using Machine Learning
}

\author{
J Sreedevi ${ }^{1}$, M Rama Bai ${ }^{2}$, G Naga Sujini ${ }^{3}$, Muthyala Mahesh ${ }^{4}$ and B Satyanarayana ${ }^{5}$ \\ 1,2,3,4 Department of Computer Science and Engineering, Mahatma \\ Gandhi Institute of Technology Hyderabad, India \\ ${ }^{5}$ Department of Mechanical Engineering, VNR Vignana Jyothi Institute \\ of Engineering and Technology, Hyderabad, India \\ Corresponding author email: jsreedevi_cse@mgit.ac.in
}

\begin{abstract}
Lung cancer is an increasing syndrome in India as well as in the world. The international agency for research says that 5.5\% of cancer patients in India are sick by lung cancer. Research is going on for predicting lung cancer detection at early stage using Computer Aided Systems. The dataset plays a important role in clinical research. If the data set contains a missed value, then we may get inaccurate results. To fill the missed values there are many number of imputation techniques. In this study Mean Imputer and KNN Imputer were used to impute the missing values. The classifiers compared are Support Vector Machine, Logistic Regression, Naïve Bayes Classifier, K-Nearest Neighbor and Decision Tree Classifier. These classifiers are compared based on accuracy. The confusion matrix is displayed for all five classifiers. At first imputation technique is applied and the accuracy of the classifiers is measured and then feature selection is done. On the selected features Principle Component Analysis (PCA) is used to increase the accuracy of the classifiers and the new accuracy of the classifiers is measured. The best combination of imputation techniques and machine learning classifiers is found to predict lung cancer. When Mean Imputation method is applied algorithms have achieved good accuracy and Decision Tree high accuracy.
\end{abstract}

KEY WORDS: IMPUTATION, CONFUSION MATRIX, PCA.

\section{INTRODUCTION}

Lung cancer is a disease that is becoming as a pandemic in India due to many reasons air pollution, tobacco consumption and many other factors. As told earlier $5.5 \%$ of cancers are lung cancer which is indicating the seriousness of the disease. By predicting the chance of cancer one can avoid it. Prevention of cancer is a tough task and also an expensive thing. American Lung Association says that 25\% of cancer deaths are due to lung cancer in 2018. World Health Organization stated in 2020 that most of the cancer deaths are due to lung cancer. We need a system that predicts the chances of lung cancer before it affects the person badly.

Biosc Biotech Res Comm P-ISSN: 0974-6455 E-ISSN: 2321-4007

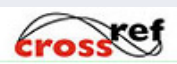

Identifiers and Pagination

Year: 2021 Vol: 14 No (5) Special Issue

Pages: $306-313$

This is an open access article under Creative

Commons License Attribn 4.0 Intl (CC-BY). DOI: $h t t p: / / d x$.doi.org/10.21786/bbrc/14.5/54
Many types of researches were done in predicting lung cancer. One way is by using CT scan images and the other way is by considering statistical data. The CT scan way of predicting is expensive when compared to statistical way because CT scan images are required in huge amount which is a difficult and expensive thing for research. By using the statistical data given by the lung cancer affected people, a try is made to construct a system that tries to predict the chances of cancer for others which is less expensive but may not be accurate as of the CT scan way of predicting. The system build takes the input and checks any missing values that are present in the data and fills the missing data. Missing values are filled with two techniques Simple imputer and KNN imputer. Then five machine learning techniques are implemented on the data and the accuracy of them is noted.

Then feature selection is done and principal component analysis is used so that the dimensionality of data is reduced and the complexity of the system decreases. The five machine learning algorithms are applied again and accuracy of them is found. The work concludes which imputation technique is best and which Machine Learning algorithm works well. The above process is applied for
Article Information

Received: $29^{\text {th }}$ Mar 2021 ccepted after revision: $27^{\text {th }}$ May 2021 
two standard datasets from the UCI repository and Data. world repository.

The authors are motivated to choose the work because of increase in number of lung cancer patients continuously. This work helps them to check the chances of lung cancer and also find the best imputation method and best Machine Learning classifier to predict the lung cancer. As the CT scan way is expensive and the statistical way of predicting this is cheap and can be easily available to every common man. Everyone can give the required data and check the chances of cancer. Working of classifiers on different datasets tested in the study. The main idea of the system built in the study is to fill the missing values with the appropriate values and apply principle component analysis on the data and feed to the machine learning algorithms and see the impact of principal component analysis on the accuracy of the machine learning classifiers and find the best Machine Learning algorithm.

A few challenges were faced during the process of study

- Datasets have only a few instances.

- Imputation methods used in the study works well only for some columns in the dataset.

- Feature selection is easy when there are few attributes but if attributes are more it is hard to select them.

- The selection comparison metric for machine learning classifier has become quite a tough task.

Measures were taken that the selected datasets have no missing values when feeding to the machine learning classifiers. If the value is filled with the wrong data type they are also considered as missing values and filled with appropriate values. The algorithms are fed with enough number of iterations so that no overfitting or underfitting occurs. Only suitable attributes are taken for prediction with no extra attributes. Many types of researches were done on predicting lung cancer and different systems were proposed. These systems were proposed based on different factors. The systems are divided based on the data they take to predict.

CT Scan Images: This system uses CT scan images of the lungs to predict the lung cancer.

These images have an area that indicates the presence of lung cancer. If the area of the lung cancer is greater than the specified threshold area then it is considered as lung cancer otherwise as no lung cancer. The images are fed to the Convolutional Neural Networks and this trained model is used to predict lung cancer. Statistical Data: This system uses the statistical data collected from different persons. This data involves different types of attributes. This type of data is required in huge amounts to train and test. The data is given to the machine learning classifiers to make predictions after training. There are many machine learning classifiers present but a suitable one is used. Data pre-processing is also a major task in this system. This system also gives good accuracy.
Many machine learning algorithms are widely. The imputation methods are not so effectively used in the preprocessing of the data. The system proposed uses two imputation methods and fills the missing data. This system finds the best one among them. After imputation principal component analysis is applied to the data then the machine learning algorithms Support Vector Machine, Decision Tree Classifier, Logistic Regression, Naïve Bayes Classifier and K-Nearest Neighbors are trained with the data, and predictions are made. The best algorithm among them is chosen based on accuracy.

\section{Objectives}

1. To find the best imputation method among Simple imputer and KNN imputer.

2. To find the best machine learning algorithm among five algorithms.

3. To reduce the dimension of the data by PCA and the complexity of the model.

4. To find the best combination of imputation method and machine learning algorithm.

Literature Survey: Lung cancer is becoming as a pandemic in the sophisticated world. Many types of research were done in the detection of lung cancer. This section deals with those researches and their modes of operations. M Sangeetha, S Mythili This system uses CNN to detect lung cancer. The input for this system is CT scan images. Preprocessing of images is done by BW Threshold. Segmentation of the image is also done then features are extracted from the pixels of images and then with this data the model is trained. The model is then used on the test dataset. Manjun BR, Athira V, Athul Rajendran In this system first feature extraction is done by Principle Component Analysis and then for classification Support Vector Machine is used. This system takes images as input. Feature extraction is done through correlation. If there is a positive correlation then it is directly proportional otherwise inversely proportional. Then SVM is used to separate these nonlinear data. The output is represented by a confusion matrix.

Negar Maleki, Yasser Zeinali, Seyed Taghi Akhavan Nikai In this system for feature selection genetic algorithm is used, and then for classification K-Nearest Neighbor classifier is used. The dataset is taken from the Data World site contains a thousand images. The missing values are imputed with the mean value of the respective column. The genetic algorithm is a 5 steps process. The performance metrics are specificity, accuracy and sensitivity. The distance in KNN classifier is measured by Minkowski distance. The accuracy attained is $96.2 \%$. Shamreen Fathima Saddique, Sharmithra P, Justin Xavier D In this study five machine learning algorithms are compared. The algorithms are Naïve Bayes, Support Vector Machine, Logistic Regression, Random Forest and K-Nearest Neighbors. The tool used is RStudio and the programming language used is R. The performance measure used is the confusion matrix. The Naïve Bayes classifier has achieved the highest accuracy of 95.24\%. 
Radhanath Patra In this study four classification algorithms are compared. The algorithms are Radical Basis Function Network, j48 Classifier, Naïve Bayes Classifier and K-Nearest Neighbors. The dataset is used is from the UCI repository. The missing value is replaced with the value that is occurring mostly in the column. The RBF classifier performs better than other classifiers with 81.25\% accuracy . Radhika P R, Rakhi.A.S.Nair and Veena $\mathrm{G}$ In this study three machine learning classifiers are compared. The algorithms are Logistic Regression, SVM, Decision Tree and Naïve Bayes Classifier. K-fold crossvalidation technique is used to split the data into test and train. The SVM has achieved the highest accuracy of 99.2\%. Dana Bazazeh and Raed Shubair In this study three classification algorithms are used. The algorithms are SVM, Random Forest and Bayesian Networks. The tool used for classification is WEKA. K-fold crossvalidation is used to split the data. The performance metrics used are precision, ROC area, accuracy and recall. Random Forest has achieved a good accuracy compared to other classifiers.

\section{System Design For MI Algorithms}

Architecture: The system consists of the following steps i) The dataset is taken and then checked if there are any missing values in the dataset. ii) The missing values are filled with imputation methods like Simple imputer and KNN imputer. The data is then divided into train and test datasets. iii) This dataset is used to train the machine learning algorithms. The accuracies of the ML algorithms are measured over a test dataset. iv) On these datasets, Principal Component Analysis is applied and this data is used to train the ML algorithms. v) The new accuracies are measured and compared with old ones. The best imputation method and machine learning algorithms will be found based on the accuracies of the systems.

Modules: The study is mainly divided into 3 distinct parts. The flow chart of the model is represented in the figure 1.

- Data Preprocessing

- PCA

- Machine Learning Algorithms

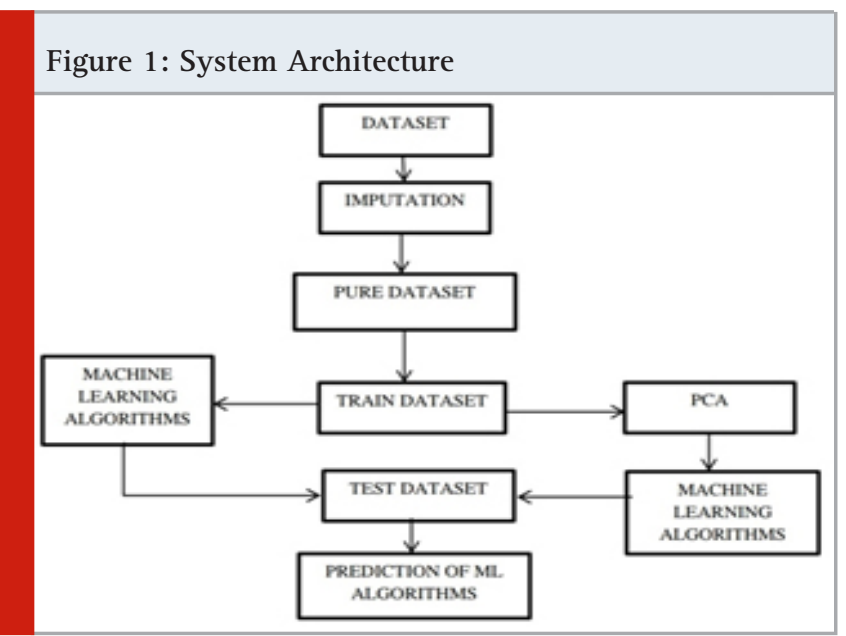

Data Preprocessing: In this section data preprocessing is done. It also includes collecting the data on lung cancer suitable for the study. Finding the missing values and filling them with approximate values. Splitting the data also plays an important role it is also done so carefully. Application of Principal Component Analysis is done on the data. By applying PCA one can reduce the complexity of the program and also the dimension of the data.

A) Data Collection: Two standard datasets are collected for the study. One dataset is collected from the UCI repository and the other dataset is collected from data. world repository. The dataset from the UCI repository has 961 instances and 6 attributes. These attributes describes the cause of lung cancer. This dataset contains some missing values in each column. This dataset contains 516 benign and 445 malignant rows. The dataset taken from the data world contains 1000 instances and 25 attributes. The attributes describe different conditions that cause lung cancer. The resulting class contains three sub-classes high, medium and low. The algorithms have to predict the person belongs to any of the three classes. The dataset is placed in Google drive so that it is easily accessible by the Google colab to run the code. The path of the dataset in the drive is specified then colab accesses it.

B) Collecting Data from CSV files: The dataset taken from different repositories is in the form of CSV files. The data in the CSV files is loaded by using the Pandas library. The function read_csv is used to load the data from a CSV files to an object. By using the object can perform any action on the data like reshaping and resizing.

Example code: data = pd.read_csv(,lung_cancer data_ world.csv').

C) Finding the missing values in the dataset: At first, any missing values are present in the dataset are checked. The dataset taken from the UCI repository has the missing values in each column. The total number of missing values in each column are noted. To check the missing values at each column there is a function isnull() which checks each value in the dataset is null or not. The sum of missing values at each column is given by data.isnull(). sum() this gives the sum.

Ex : data.isnull().sum()
column_a 2
column_b 5
column_c 31
column_d 48
column_e 76
column_f 0
dtype: int64.

D) Finding the missing values with different data type: The function used above has a limitation. It only checks any missing value is present or not but doesn't check any different type of object is present in the dataset. For example, a column support integer data type if there present any string data type the above function it doesn't count so to mark such values as missing values those have to be mentioned explicitly. The explicitly mentioned words are checked with each value in the dataset if any matching is found then they are marked as a missing 
value. Otherwise, consider it as a valid value.

E) Filling the missing values in the dataset: There are three ways of filling the missing values.

i) One way is by dropping the columns with the missing values. This technique is used for datasets with a huge amount of data that is datasets having more than ten thousand rows. This can be done by using the dropna() function. By this, the columns with missing values can be dropped.

ii) The second way is statistical one in this the statistical methods are used to fill the data. The statistical methods include mean, median, mode (most_frequent) and constant value. In the Mean method, the mean value of the column is placed in the place of the missing values. In the median method, the median value which is the middle value of all the values in the column is placed at the missing place. In mode or most frequent method, the value that is occurring more number of times is placed in the place of missing value. In the constant method, the constant value or the fixed value is placed in the place of missing value. In this study, the Mean method is used to fill the values.

Ex: SimpleImputer ( $m$ issing_values $=n p$. nan,strategy='mean',verbose=0) \# ['mean', 'median', ' most_frequent', 'constant']

s_imputer.fit(data.iloc[1:,0:5]) \# fit function fits the data with missing values

s_imputer.transform(data.iloc[1:,0:5]) \# the transform function fills the missing values with the appropriate statistical value.

iii) The third way of filling the missing value is constructing a new model that fills the missing values. In this technique, the rows without missing values are treated as train data and rows with missing values are treated as test data. The missing values are predicted. All the columns except the missing value column are treated as input attributes and the missing value column is treated as output attribute. In this way, all the columns with the missing values are filled. Some techniques are Iterative Imputer and KNN Imputer. In this study, the KNN Imputer is used. In the sklearn library, there is a module impute by which the KNNImputer module can be imported by which imputation can be performed. The KNN imputer works the same as the KNN algorithm. In this technique we specify the number of neighbors to be checked to fill the value. The imputer first finds the nan_euclidean_distances between the tuple with the missing value and tuples with no missing value tuples. The tuples which are having less distance are taken and the column with the missing value is filled with the average value of the selected short distance tuples of a specified number. In this way, all the missing values in the dataset are filled.
Ex: KNNImputer(n_neighbors=10) \#KNN finds ten most similar tuples by nan_euclidan distance and finds the average the value of ten tuples. impute_knn=impute knn.fit(data.iloc[1:,0:6]) data.iloc[1:,0:6]=impute_knn. transform(data.iloc[1:,0:6])

The output is the dataset with filled values.

In this study SimpleImputer which is the Mean method and KNNImputer are used and the best imputation method among both is found.

F) Feature Selection: In the datasets selected for the study, one has 6 attributes and the other has 25 attributes. All the attributes may not be useful and also some of the attributes may be dependent on one another. To remove the attributes that are not useful and dependent on one other the process called feature selection is used as shown in Figure 2. Feature selection can be either explicit selection or calling a function to select the attributes based on some model. In explicit selection, the attributes necessary for the prediction of lung cancer can be specified on observation or importance. The function also selects the attributes necessary for the prediction of lung cancer.

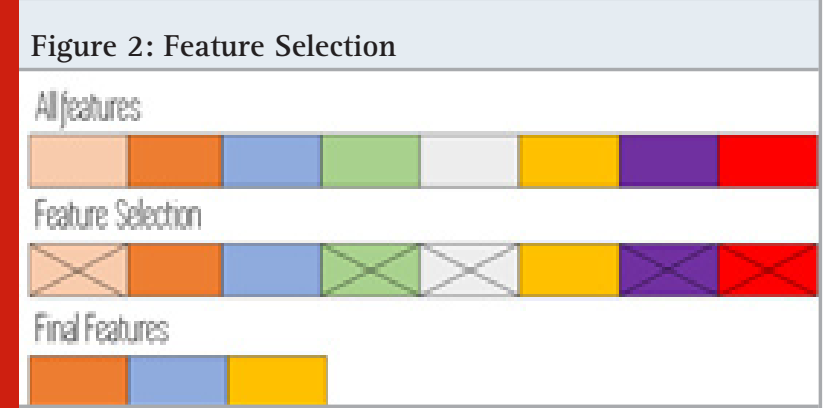

Figure 3: PCA

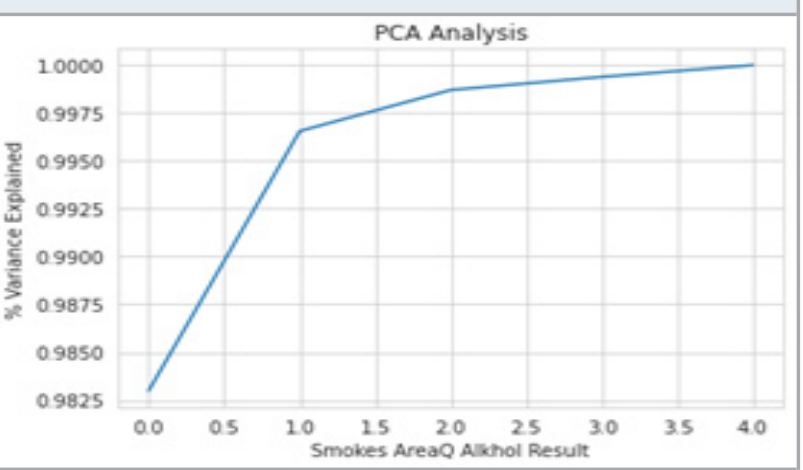

The feature selection model used in the study is Select From Model. In this technique, the estimator assigns importance to attributes and specifies some threshold if the value of the specific attribute is below the threshold the attribute is discarded otherwise the attribute is selected. This method has two ways of selecting the features L1 based and Tree-based feature selection. In this study L1 based feature selection is used. The Logistic regression and LinearSVC are used for classification 
with these the sparsity is controlled. The larger the sparsity more features are selected. Sklearn has modules LinearSVC and SelectFromModel by which the feature selection can be made.

Figure 4: Logistic Regression

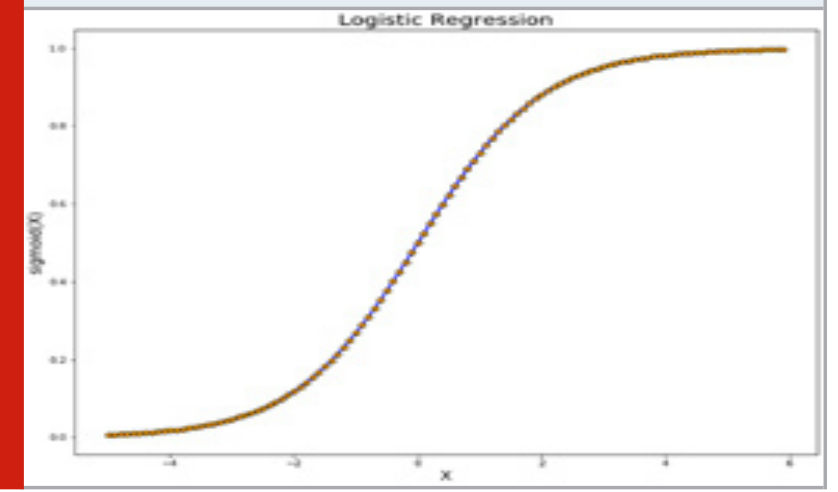

Ex: LinearSVC(C=0.5,).fit(A,B) SelectFromModel(lsvc, prefit=True) transform(Z).

G) Principal Component Analysis: It is used for reducing the dimensions of the data. The data is projected onto the lower dimension. PCA is done in 3 steps. In step one is standardization of the given data. In step two computation of the covariance matrix is done so that we can find is there any relation between the attributes. In step three eigenvectors and values of the covariance matrix are computed by this the principal components are found. In this way, the important features are selected and complexity is reduced. In this study, the PCA module is used from the sklearn library decomposition module shown in figure 3.

H) Splitting the Dataset: Each dataset taken for the study is divided into train and test datasets. The training data is $80 \%$ of the data and for testing $20 \%$ of the data is used. The dataset is divided in such a way that the result attribute has equally divided the classes malignant and benign. This process is done by the train_test_split module in sklearn Library.

Machine Learning Algorithms: In the study done here, five machine learning algorithms are used. These algorithms are trained and tested over the dataset and compared. The imputation methods are discussed before in this section the working of the machine learning algorithms is discussed.

i) Logistic Regression: It is a classifier that separates the data linearly. With logistic regression, the data can be linearly separated. In this study, the logistic Regression used is imported from sklearn. To train the model fit() function is used. To predict target values predict() function is used. The logistic Regression is a sigmoid function as shown below in figure 4 . The formulae of the logistic function are represented below

$\mathrm{h}(\mathrm{x})=\operatorname{sigmoid}(\mathrm{Z})$
Sigmoid $=1 /\left(1+e^{\wedge}-x\right) X=w 0+w 1 \times 1+w 2 \times 2 \ldots+w n x n+b$

Where wi $=$ weights, $x i=$ input and $\mathrm{b}$ is bias.

By changing the $w$ and $b$ values the curve can be fit and make the classification required. By changing the value of $b$ the curve can be moved left or right on the $\mathrm{x}$-axis. By changing the value of the $\mathrm{w}$ the slope of the curve can be changed as required to make the classification of the data.

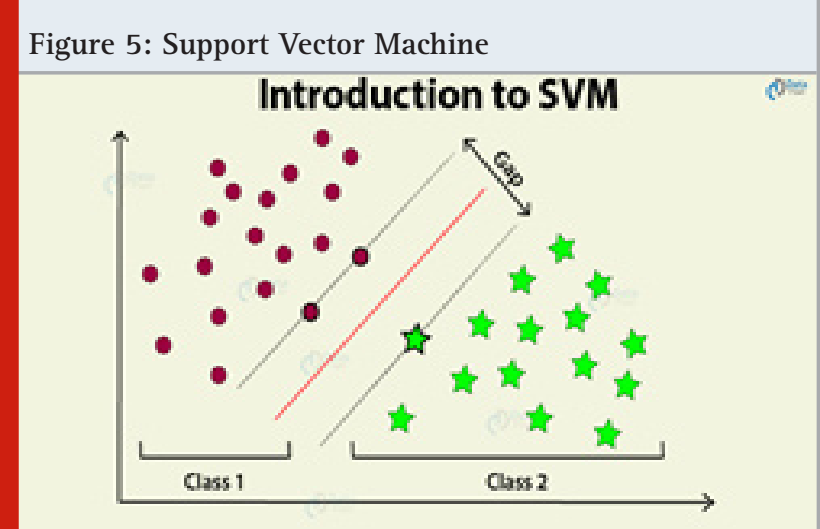

Figure 6: Naïve Bayes

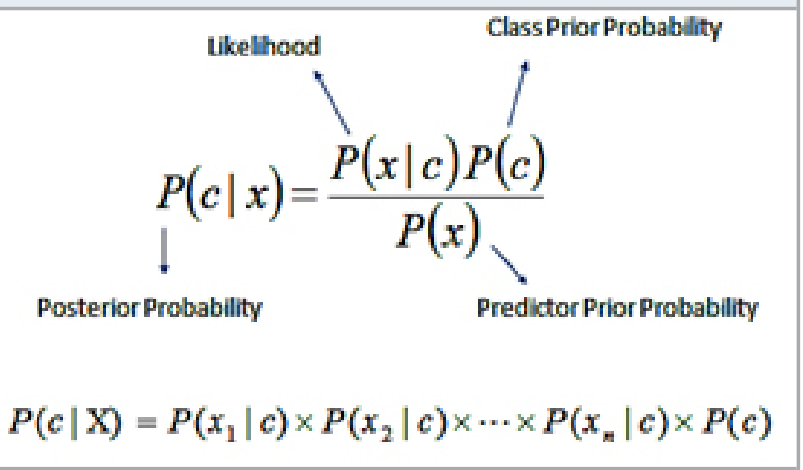

ii) Support Vector Machine: Support vector machine is a classification algorithm that separates the data into two groups. The lung cancer data is divided into groups and classification is done as patient as cancer or not. It creates two hyperplanes one is close to positive points and the other to the negative points. The middle line is the margin line which helps in classifying the data as shown in figure 5 . Margin line $(\mathrm{y})=\left(\mathrm{W}^{\wedge} \mathrm{T}\right)^{*} \mathrm{X}+\mathrm{b}$ where $\mathrm{W}$ is the slope of the line, $\mathrm{X}$ is input and $\mathrm{b}$ is the intercept. In this study, OneVsRestClassifier is used by taking one group and classify it against other groups. The fit function is used to train and predict is used to predict the values. Support vector machine helps in differentiating the data into groups. In binary classification, two groups are separated easily but in multi-class classification, one group is separated and predicted over other classes treating them as one class. The second dataset taken from data world has three classes.

iii) Naïve Bayes Classifier: It is a probabilistic machine learning algorithm. It works on the Bayes theorem. 
It predicts that each row of data in the given dataset belongs to the particular class by calculating the probability of data of each class. The class with the highest probability is chosen and the respective class is predicted. $\mathrm{P}(\mathrm{c} \mid \mathrm{x})$ is the probability of data to belongs to that class the formulae is shown in figure 6. In this study, GaussianNB is used. It works for the continuous values, follows the normal distribution. The fit function trains data and predicts the function predicts the data.

iv) Decision Tree Classifier: The decision tree is a tree-like structure. The root node indicates the resultant class. The internal node indicates the conditions which lead to the root nodes that are to the resultant class. The decision is a set of if-else statements where several if-else statements are put together as shown in figure 7 in the form of a tree. This set of statements checks the values and form conditions. This if-else 17 condition seems to be easy but it requires a lot of work to put such statements together to make a classifier. In this study, the function used is DecisionTreeClassifier. The Decision tree classifier is a process where tree-like if-else statements are marked. The fit and predict function train and predict the output.

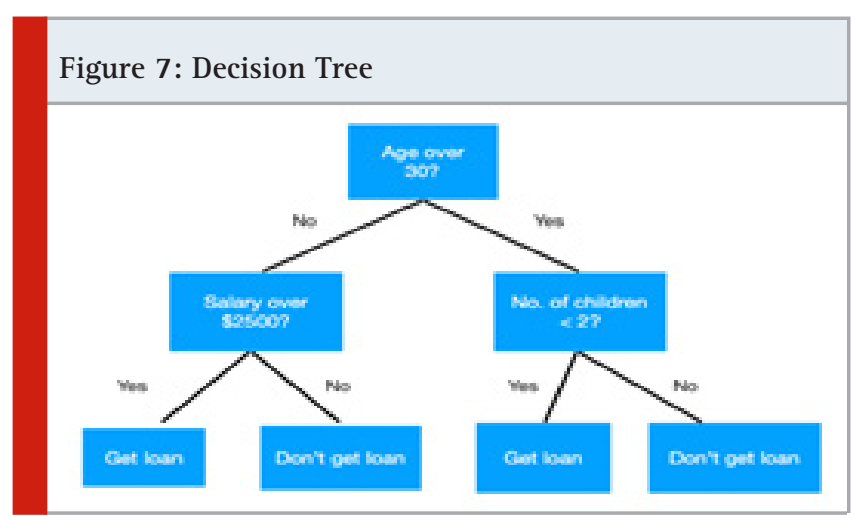

Figure 8: Nearest Neighbor

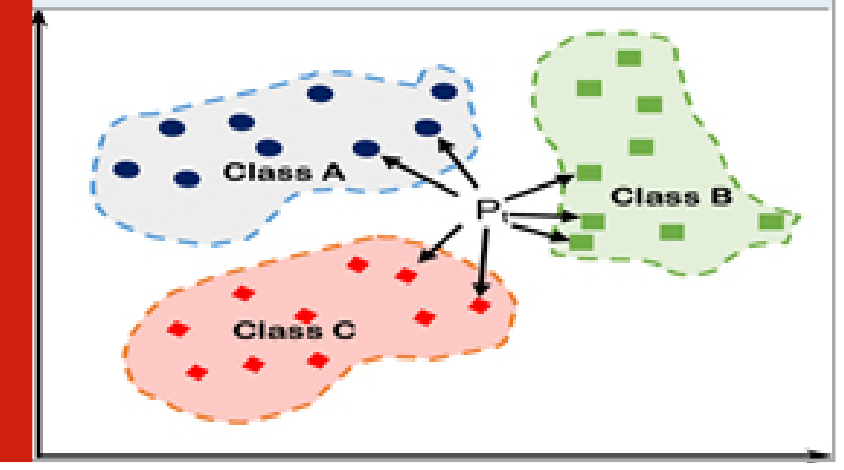

v) K-Nearest Neighbor Classifier: It classifies the data by the measure of similarity between the data. This algorithm divides the data into groups and forms the groups into different classes by giving them class labels as shown in figure 8. Initially, the number of neighbors is given along with some classes. Then the algorithm divides the data into groups by iterating on the data by measuring the distance between the data points. The points assigned to the class with the least distance from them. The distance measurement can be Manhattan or
Euclidian distance. In this study, the function used is the KNeighborsClassifier function. The fit function trains the model and the predict function predicts the output.

Confusion Matrix: Confusion Matrix is the 2D box shown in figure 9 that contains four separate classes the classes are defined as True positive if the output predicted and output of original class is true then this section increases the count by 1 . False Positive if the output predicted is true and the original output is false then this section increases the count by 1 . False Negative if the output of the predicted class is false and the output of the original class is true then this section increases the count by 1. True Negative if the output of the predicted class is false and the output of the original class is also false then this section increases count by 1 . By using these four parameters we calculate accuracy, recall, precision, sensitivity and other factors. The confusion matrix is calculated for all five algorithms in the study.

Figure 9: Confusion Matrix

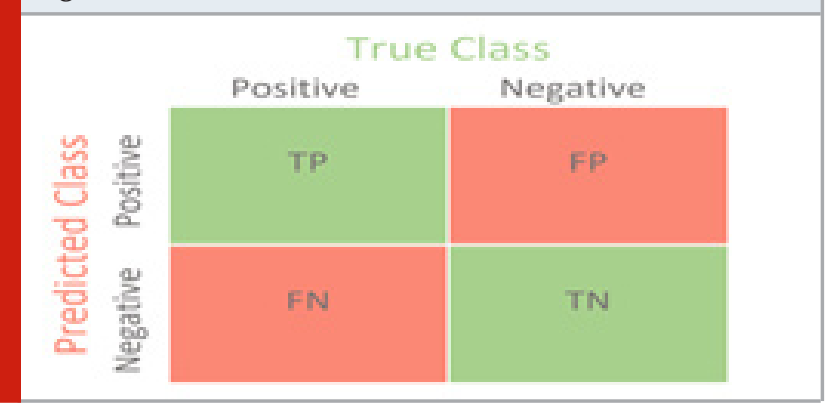

Training and Testing: In this work, after preprocessing of the data which includes filling the missing values, the data is then sent to the training and testing part of the study. Before training and testing the data is split into train and test part. Then the data is given to the machine learning algorithms and the accuracy of all the five algorithms is noted then the feature selection is applied to the data and required features are selected. The principal component analysis is applied to the dataset. This new dataset is sent to machine learning algorithms and the new accuracy of the algorithms is noted.

\begin{tabular}{|c|c|c|}
\hline & KNN & Mean \\
\hline column_c & $\begin{array}{l}2.6 \\
3.3\end{array}$ & 2.72 \\
\hline column_d & $\begin{array}{l}1 \\
2.8\end{array}$ & 2.79 \\
\hline column_e & $\begin{array}{l}2.7 \\
3\end{array}$ & 2.91 \\
\hline
\end{tabular}

Training: Two imputation methods applied on the dataset the Simple Imputer and KNN Imputer. The five machine learning algorithms Logistic regression, Naïve Bayes Classifier, Decision Tree Classifier, K-Nearest Neighbor and Support Vector Machine are trained with 
the preprocessed dataset. The respective fit() function is used to train the algorithms.

\section{Testing}

Imputation Methods: Imputation of the data by two methods is shown in table 1 the point to be noticed is the KNN Imputer fills the missing values with different values but Mean Imputer fills the data with only one value. KNN imputer takes the average value of specified number of similar tuples. Similar tuples are found with the nan_eculidian distance. So the value changes from tuple to tuple based on similar tuples. As shown in table 1 for column_c the KNN imputer puts the values of 2.6 in one tuple and 3.3 in the other tuple. In column_d and column_e, the same process is repeated. Only two tuples are taken as an example the process works similar for all the missing value tuples. The Mean imputer puts the same value in all the missing value tuples of respective columns.

\begin{tabular}{|c|c|c|c|c|c|}
\hline & Logistic Regression & SVM & Naïve Bayes & Decision Tree & $\mathrm{K}-\mathrm{NN}$ \\
\hline Before PCA & 0.84 & 0.81 & 0.79 & 0.75 & 0.78 \\
\hline $\begin{array}{l}\text { Mean Imputer } \\
\text { After PCA (a,b,e,f) }\end{array}$ & 1 & 0.98 & 1 & 1 & 0.93 \\
\hline Before PCA & 0.83 & 0.81 & 0.79 & 0.77 & 0.78 \\
\hline $\begin{array}{l}\text { KNN Imputer } \\
\text { After PCA (a,b,d,e,f) }\end{array}$ & 1 & 0.97 & 1 & 1 & 0.89 \\
\hline
\end{tabular}

Table 3. Comparison of ML algorithms on Data world dataset

\begin{tabular}{|l|c|c|c|c|c|}
\hline & Logistic Regression & SVM & Naïve Bayes & Decision Tree & KNN \\
\hline $\begin{array}{l}\text { Before PCA } \\
(26 \text { features })\end{array}$ & 0.97 & 1 & 0.91 & 1 & 1 \\
\hline $\begin{array}{l}\text { After PCA } \\
(23 \text { features })\end{array}$ & 1 & 1 & 1 & 1 & 1 \\
\hline
\end{tabular}

Figure 10: Confusion Matrix of all five algorithms

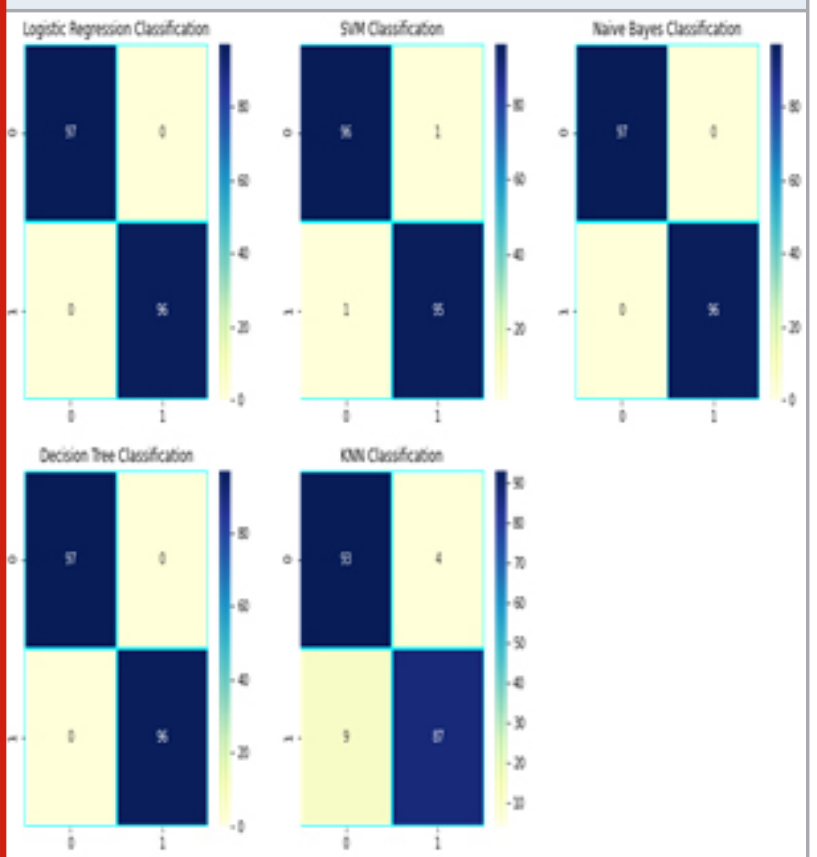

Machine Learning Algorithms: The comparison of results of Machine Learning Algorithms is shown in table 2 is for the dataset taken from the UCI repository. The five
ML algorithms not achieved good accuracy before the application of PCA. Logistic Regression has achieved an accuracy of 84 percent on the dataset with Mean imputation applied to it. Mean imputation works well before PCA is applied. After application of PCA Logistic regression, Naïve Bayes and Decision Tree has achieved an accuracy of 100 percent in both imputation methods. KNN achieved an accuracy of 89 percent least of all the five algorithms. PCA has changed the efficiency of the algorithms a lot. By PCA the complexity of the dataset also decreased. Only 4 features are used after feature selection for PCA they are BI-RADS assessment, Age, mass density, Severity.

The working of algorithms on data world dataset has a quite different role as shown in Table 3. SVM, Decision Tree and KNN have achieved 100 percent accuracy without the application of PCA. After the application of PCA, all the five algorithms have achieved 100 percent accuracy by using 23 features among 26 . The confusion matrix of all the algorithms on the UCI dataset is shown in figure 10. The numbers shown are in percentage of data belongs to that category may be True Positive, False Positive, True Negative and False Negative.

\section{CONCLUSION AND FUTURE SCOPE}

The study aimed to find the best imputation method and 
best machine learning algorithm. The Mean imputation technique is effective compared to KNN imputation technique. This conclusion was drawn because the accuracies of algorithms in Mean imputation method are better than KNN imputation method. Before application of Feature selection and PCA the five algorithms had low accuracy. After application of feature selection and PCA the algorithms performed well on both the datasets. The five algorithms have achieved 100\% accuracy on Data world dataset. The algorithms Logistic Regression, Naïve Bayes and Decision Tree have achieved 100\% accuracy on the UCI dataset. When comparing both the datasets Decision Tree works well. Naïve Bayes and Logistic Regression also give good results. The study, at last, concludes that Mean Imputation for missing values with the Decision Tree algorithm gives good achievable results.

\section{REFERENCES}

Ada, Rajneet Kaur (2013), "A Study of Detection of Lung Cancer Using Data Mining Classification Techniques", International Journal of Advanced Research in Computer Science and Software Engineering, Volume 3, Issue 3, March.

D. Delen, G. Walker, A. Kadam, (2005) "Predicting breast cancer survivability: a comparison of three data mining methods", Artificial Intelligence in Medicine, Vol.34. Dana Bazazeh, Raed Shubair,( 2016) "Comparative Study of Machine Learning Algorithms For Breast Cancer Detection And Diagnosis", 5th International Conference On Electronic Devices, Systems And Applications (ICEDSA).

Gurcan, M. N, Sahiner, B, Petrick, N, Chan, H.-P, Kazerooni, E. A., Cascade, P. N, Hadjiiski, L, $(2002$ ) "Lung nodule detection on thoracic computed tomography images: Preliminary evaluation of a computeraided diagnosis system”, Medical Physics. Janee Alam, Sabrina Alam, Alamgir Hossan,( 2018) "Multi-Stage Lung Cancer Detection and Prediction Using Multi-class SVM Classifie”, International Conference on Computer, Communication, Chemical, Material and Electronic Engineering (IC4ME2).

K. Kourou, T. P. Exarchos, K. P. Exarchos, M. V. Karamouzis, D. I. Fotiadis, (2015) "Machine learning applications in cancer prognosis and prediction", Comput. Struct. Biotechnol Journal vol 13.

M Sangeetha, S Mythili, (2021) "Detection and characterization of lung cancer Tumor by using convolutional neural network", Journal of Physics: Conference Series, Volume 1717.

Mandhir Kaur, Dr. Rinkesh Mittal,( 2014) “Survey of Intelligent Methods for Brain Tumor Detection”, IJCSI International Journal of Computer Science Issues, Vol. 11, Issue 5, September.

Manjun BR, Athira V, Athul Rajendran, (2021 ) "Efficient multi-level lung cancer prediction model using Support Vector Machine classifier", IOP Conference Series: Materials Science and Engineering, Volume 1012,.

MS. Bhagyashree, Akshay Pancha, Dilip Chavan, "Lung cancer detection using deep learning”, 2nd International Conference on Advances in Science \& Technology (ICAST-2019).

Negar Maleki, Yasser Zeinali, Seyed Taghi Akhavan Nik A KNN method for lung cancer prognosis with the use of genetic algorithm for feature selection", Expert Systems with Applications, sept 2020.

Radhanath Patra,(2020) "Prediction of lung cancer using machine learning classifier”, International Conference on Computing Science, Communication and Security, july.

Radhika P R, Rakhi.A.S.Nair And Veena G, (2019 ) "A Comparative Study Of Lung Cancer Detection Using Machine Learning Algorithms", IEEE International Conference On Electrical, Computer and Communication Technologies (ICECCT).

Shamreen Fathima Saddique, Sharmithra P, Justin Xavier D,( 2018) "Prediction of lung cancer using classifier models", International Journal of Science and Research (ijsr), volume 7 issue 3, march.

Yuksel Oner, Taner Tunc, Erol Egrioglu, Yildiz Atasoy, (2013) "Comparisons of Logistic Regression and Artificial Neural Networks in Lung Cancer Data”, American Journal of Intelligent Systems, Vol. 3 No. 2. Z.Karhan, T.Tunc, "Lung Cancer Detection and Classification Algorithms”, IOSR Journal of Computer Engineering (IOSR-JCE), Volume 18, Issue 6, Ver. III (Nov.-Dec. 2016). 